Awarding change:

Maria Freire reflects

on science with

a global impact

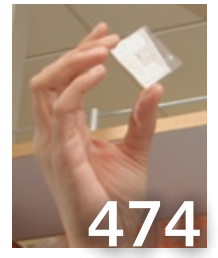

Hot chip:

Microfluidics heats up

thanks to help from a

toaster oven

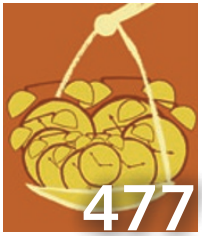

Alarm call:

New clues hint at how

sleep loss might spell

weight gain

\title{
Return to the basics might breathe life into HIV vaccine pipeline
}

As the financial world grapples with major market corrections, the HIV research community faces its own reality check. The scientific reassessment comes after disappointing results from a candidate HIV vaccine developed by Merck caused the company to halt human trials of the potential vaccine last September.

"There's no question in my mind that the vaccine research effort is in need of a major midcourse correction," Warner Greene of the University of California, San Francisco told listeners at a government-sponsored summit on HIV vaccine research on 25 March in Bethesda, Maryland. Greene, who co-chaired the summit, emphasized the need for a return back to the basics to, for example, create better animal models for testing vaccines before racing ahead with numerous human clinical trials.

Talking about the need for more basic HIV vaccine research is easy, but figuring out how to fund more of such experiments is not. The US National Institute of Allergy and Infectious Diseases currently devotes $\$ 225$ million a year to basic HIV vaccine discovery research. Still, at the recent summit, Anthony Fauci, who leads the institute, estimated that the country's flat research budget has over the past five years led to a $15 \%$ decline in purchasing power to support scientific projects. This decrease, he noted, comes at a time when "we have challenges in that there are so many things that we do not know in this field of HIV vaccine [development]." Fauci added that redirecting current funds and starting new initiatives might make more money available for basic research in this area.

If extra money does materialize, experts say this could help support studies into vaccine dose and delivery methods. It could also bolster bold departures from previous attempts to develop an HIV vaccine. In particular, many scientists hope to accelerate experiments examining the protective effect of vaccines based on replicating viral 'vectors' engineered to produce immunity by shuttling foreign genetic information into cells. "Everybody's started talking about replicating vectors," says Ben Berkhout at the University of Amsterdam in the Netherlands.

Part of the inspiration for working with replicating vectors comes from the fact that, thus far, the best protection against simian

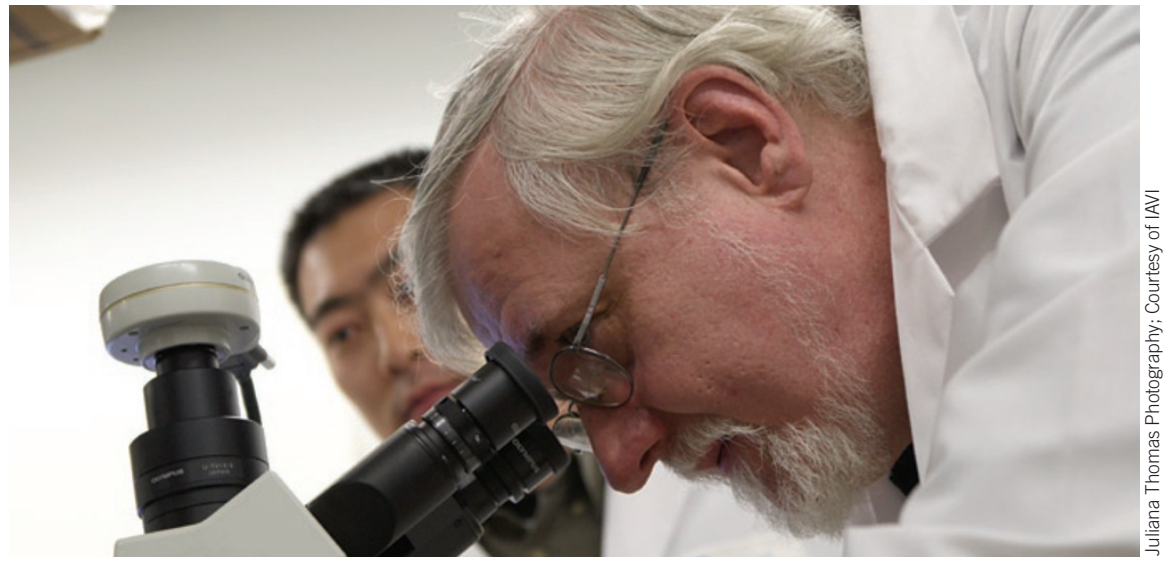

A closer look: Timothy Zamb of IAVI and collaborators consider new HIV vaccine options

immunodeficiency virus (SIV, a virus similar to HIV that infects monkeys) in animal tests has come from vaccines based on a weakened but still replicating form of SIV itself.

The hope is that so-called 'live' vectors, which rely on harmless viruses, might induce a stronger immune response than vectors lacking the ability to replicate (such as the one used in the recently halted Merck trial). One scientist exploring this approach is Louis Picker at the Oregon Health \& Science University in Beaverton, Oregon. His team has engineered replicating versions of the cytomegalovirus (CMV)—a generally harmless microbe that infects approximately $80 \%$ of people worldwide- to contain different genes from SIV. Testing to see if these CMV-based vaccines protect against SIV began in late March.

\section{Exploring options}

Timothy Zamb, who heads the vaccine research and development laboratory of the International AIDS Vaccine Initiative (IAVI) in New York, says that scientists need to explore a variety of vectors-including both those that can replicate and those that cannot- to optimize vaccine options for the future. Beyond providing funding, IAVI has also helped organize a consortium of researchers focused on vector design to support this effort.

But predicting the promise of viral vectors remains a tricky task because they can induce different types of immune responses, according to Berkhout: "One virus that replicates is not the same as another that replicates."

Some experts worry about potential hurdles in product approval for vaccines based on replicating vectors. Still, Picker stresses that this approach could yield insights into how to block HIV infection: "This isn't about the regulatory issues. This is about the basic science to see what immune response works."

Other scientists have meanwhile focused on designing candidate HIV vaccines based on viral vectors capable of only one cycle of replication-which are considered potentially safer than a fully live vaccine. John Rose, a virologist at the Yale University School of Medicine in New Haven, Connecticut, and his colleagues have engineered a way to limit the replication of the vesicular stomatitis virus to one cycle. "We decided we had to work on single-cycle vectors because they would be given a green light more quickly," says Rose, referring to potential regulatory hurdles.

And although scientists generally say that a vaccine based on live, attenuated HIV is currently "not on the table" as a viable option in humans, the effort to further develop singlecycle attenuated SIV continues (J. Virol. 78, $11715-11725 ; 2004)$. So while the prospect of more funds for basic discovery research into HIV vaccines has gained general approval from laboratory researchers, the discussion about the best path to a vaccine remains a live debate.

Roxanne Khamsi, New York 